\title{
Analysis of Adherence to Acute Inpatient Rehabilitation in Patients with Cancer
}

\author{
Jegy M. Tennison, MD ${ }^{\circledR}$; Carly M. Sullivan, OTR²; Brian C. Fricke, MD³; Eduardo Bruera, MD ${ }^{1}$ \\ 1. Department of Palliative, Rehabilitation, and Integrative Medicine, The University of Texas MD Anderson Cancer Center, Houston, Texas. \\ 2. Department of Rehabilitation Services, The University of Texas MD Anderson Cancer Center, Houston, Texas. \\ 3. Department of Rehabilitation Medicine, UT Health San Antonio Long School of Medicine, San Antonio, Texas.
}

$\triangle$ Corresponding author: Jegy M. Tennison, MD, Department of Palliative, Rehabilitation, and Integrative Medicine, Unit 1414, The University of Texas MD Anderson Cancer Center, 1515 Holcombe Boulevard, Houston, TX 77030. Phone: (713) 745-2327; fax: 713-792-6092; E-mail: jmtennison@mdanderson.org.

() The author(s). This is an open access article distributed under the terms of the Creative Commons Attribution License (https://creativecommons.org/licenses/by/4.0/). See http://ivyspring.com/terms for full terms and conditions.

Received: 2021.03.29; Accepted: 2021.07.29; Published: 2021.08.20

\begin{abstract}
Importance: The need for cancer rehabilitation is expected to continue to dramatically increase with the aging population and increasing number of cancer survivors. These survivors experience a wide range of physical limitations and symptoms that negatively affect their health and quality of life. Research is needed to determine the rate of adherence, reasons for non-adherence, and interventions to improve adherence to acute inpatient rehabilitation among patients with cancer.

Objective: To evaluate the rate of adherence and reasons for non-adherence to acute inpatient rehabilitation in patients with cancer.

Design, Setting, and Participants: This was a secondary analysis of a retrospective study that assessed medical complications in 165 patients with cancer who had a median length of stay of 11 days (interquartile range of 8-14) in acute inpatient rehabilitation. We reviewed the records of all consecutive patients who underwent acute inpatient rehabilitation from September 1, 2017 through February 28, 2018 at a large academic, quaternary National Cancer Institute-designated Cancer Center.

Main Outcomes and Measures: We calculated the rehabilitation session adherence rate and descriptively summarized the reasons for non-adherence.

Results: There were $78 / 165$ (47\%) patients that had 1 or more incomplete rehabilitation sessions due to medical complications. These patients had a median of 2 (interquartile range of 1-4) incomplete rehabilitation sessions. We noted other reasons for incomplete rehabilitation sessions in 146/165 (89\%) patients, who had a median of 3 (interquartile range of 2-4) incomplete rehabilitation sessions. The median total number of days with incomplete rehabilitation sessions in the entire cohort was 2 (interquartile range 1-3).

Conclusion and Relevance: Among patients with cancer undergoing acute inpatient rehabilitation, the adherence rate to 1-hour long intensive rehabilitation sessions were low due to medical complications and other reasons. This in turn affected compliance with the 3 hours of rehabilitation a day requirement for acute inpatient rehabilitation. Patients with cancer undergoing acute inpatient rehabilitation are medically complex and further research at multiple institutions with larger cohorts may be beneficial in further assessing adherence rates and reasons for non-adherence to improve participation in acute inpatient rehabilitation.
\end{abstract}

Key words: neoplasm, inpatients, guideline adherence, exercise, occupational therapy, rehabilitation

\section{Introduction}

For admission to acute inpatient rehabilitation, the Centers for Medicare \& Medicaid Services (CMS) require that the patient 1 ) undergo an intensive rehabilitation program generally consisting of 3 hours of therapy per day at least 5 days a week with multiple therapy disciplines; 2 ) is medically stable 
and expected to benefit significantly, with measurable functional improvement from active participation within a prescribed period of time; 3 ) is supervised by a rehabilitation physician with face-to-face visits at least 3 days a week; and 4) undergoes the program with a coordinated, interdisciplinary team approach [1]. These guidelines are mandatory for CMSaffiliated payors and other payors tend to follow them. The goal of acute inpatient rehabilitation is to provide concurrent medical and rehabilitative care for patients [2].

In a systematic review, the adherence rate for supervised physical therapy in outpatient settings for patients with cancer was greater than $64 \%$ [3]. There is a gap in knowledge regarding adherence to acute inpatient rehabilitation in patients with cancer, especially related to medical complications, which have been noted to be high at our institution [4]. Therefore, the purpose of this study was to assess adherence and describe the reasons for not participating fully during acute inpatient cancer rehabilitation to pioneer this topic in the medical literature.

\section{Methods}

This is a secondary analysis of a retrospective study that included 165 patients, whose demographic and clinical characteristics along with data collection procedures were described and published previously (see Table S1) [4]. Approval for data collection was obtained from the institutional review board.

Progress notes from rehabilitation clinicians (physical therapists, occupational therapists, speech \& language pathologists, physical medicine \& rehabilitation physicians, and their advanced practice providers) were reviewed to measure adherence and identify reasons for incomplete therapy sessions, which were defined as fewer than 45 minutes of participation out of 60 minutes scheduled per rehabilitation session. At our institution, the rehabilitation sessions are provided as 1-hour sessions 3 times a day on weekdays and an additional 1-hour session on a weekend day. Sessions on weekends, holidays, admission days, and discharge days were excluded from this study because patients did not consistently undergo 3 hours of rehabilitation sessions on those days. The duration of each rehabilitation session is documented in each progress note by physical and occupational therapists. Speech and language pathology sessions were assumed to be complete sessions (unless explicitly stated as a missed session) as their durations were not recorded.

We assessed adherence by measuring the frequency and proportion of patients who were noted to have 1 or more incomplete rehabilitation sessions on days when they should have had 3 hours of rehabilitation sessions. We did not assess adherence based on the total number of rehabilitation sessions since patients had an additional rehabilitation session on a weekday $\left(6^{\text {th }}\right)$ day beyond the requirement of 3 hours of rehabilitation sessions per day at least 5 days out of the week. In identifying reasons for incomplete rehabilitation sessions, problems identified as moderate or high risk of complications in the medical decision-making section of CMS guidelines [5] were defined as medical complications [4]. The details regarding these complications were described previously [4]. Data were managed using Research Electronic Data Capture for descriptive statistical analysis.

\section{Results}

The total cohort of 165 patients had a median length of stay of 11 days (interquartile range of 8-14) in acute inpatient rehabilitation. Among these patients, there were $61(37 \%)$ patients who had metastatic solid tumors and 69 (42\%) who had solid tumors without metastasis. Baseline demographic and clinical characteristics including neoplasm types were previously published (see Table S1) [4]. There were 91 (55\%) surgical admission patients and 74 (45\%) medical admission patients. These patients were transferred to acute inpatient rehabilitation due to neoplasm and/or its treatment-related deconditioning and neuromuscular deficits. Comorbidities included the following: hypertension 80 (49\%), diabetes mellitus 36 (22\%), depression 32 (19\%), obesity $21(13 \%)$, chronic pulmonary disease 17 $(10 \%)$, renal failure $15(9 \%)$, congestive heart failure 7 $(4 \%)$, liver disease $4(2 \%)$, peripheral vascular disease $3(2 \%)$, peptic ulcer disease $3(2 \%)$, and rheumatologic disease $1(0.5 \%)$.

Seventy-eight out of 165 (47\%) patients had 1 or more incomplete rehabilitation sessions due to medical complications (Table 1), with a median of 2 (interquartile range 1-4) incomplete sessions. Most of the medical complication management involved symptom management 93 (39\%), with interventions for pain management $47(20 \%)$ being most frequent. We noted other reasons for incomplete rehabilitation sessions in $146(89 \%)$ patients (Table 2) with a median of 3 (interquartile range of 2-4) incomplete rehabilitation sessions. Of these other reasons for incomplete rehabilitation sessions, the most frequent were due to patient-reported symptoms 158 (32\%), with fatigue 115 (24\%) being most frequent. The median total number of days with fewer than 3 hours of rehabilitation sessions was 2 (interquartile range 1-3) in the entire cohort. 
When patients are transferred from acute medical care to acute inpatient rehabilitation, their medical condition should be stable [1] and they should be able to tolerate 3 hours of rehabilitation daily to prevent readmission to acute medical care services [6]. Although some studies [4, 7-9] have evaluated risk factors for return to acute care services and our previous study have evaluated medically unstable conditions [4] during acute inpatient rehabilitation in patients with cancer, further studies are needed to delineate the various and extensive unstable medical conditions as well as other reasons that prevent full participation during acute inpatient rehabilitation. Besides treatment time, the content of rehabilitation activities and patients' tolerance to those activities are important factors to be considered when evaluating overall functional gain.

\section{Limitations}

This study is limited by its small sample size and assessment at a single institution. Because this was a retrospective study, it depended on the accuracy of the rehabilitation clinicians' progress notes. Factors such as the phase of oncological treatment, patients' motivation or level of education, and living conditions were not documented consistently in the majority of the studied population before transfer to acute inpatient rehabilitation. Unlike physical and occupational therapy progress notes, speech and language pathology progress notes did not routinely document session duration. Therefore, we could have underestimated the true rate of adherence and overall assessment of various reasons for non-adherence. Nevertheless, this pilot study provides a framework for understanding adherence to acute inpatient rehabilitation.

\section{Conclusion}

Among patients with cancer undergoing acute inpatient rehabilitation, the number of patients unable to adhere to 1-hour long rehabilitation sessions to complete 3 hours of rehabilitation sessions a day was high. Further research is needed to assess adherence and reasons for non-adherence and improve participation to maximize functional gains from acute inpatient rehabilitation.

\section{Abbreviations}

CMS: Centers for Medicare \& Medicaid Services.

\section{Supplementary Material}

Supplementary table S1.

http://www.jcancer.org/v12p5987s1.pdf

\section{Acknowledgements}

We thank the data management team at the Department of Palliative, Rehabilitation \& Integrative Medicine and editing services in the Research Medical Library at the University of Texas MD Anderson Cancer Center.

\section{Ethical approval statement}

This study was approved by the institutional review board.

\section{Authorship}

- Conception and design: Jegy Tennison, Eduardo Bruera;

- Administrative support: Eduardo Bruera;

- Acquisition of data: Jegy Tennison, Carly Sullivan, Brian Fricke;

- Data analysis and interpretation: Jegy Tennison, Eduardo Bruera;

- Manuscript writing and final approval: All authors.

\section{Competing Interests}

The authors have declared that no competing interest exists.

\section{References}

1. Centers for Medicare \& Medicaid Services. Inpatient Rehabilitation Facility (IRF) Medical Review Changes. 2018. https://www.cms.gov/Outreach-andEducation/Medicare-Learning-Network-MLN/MLNMattersArticles/Downl oads/SE17036.pdf.

2. Boldt $\mathrm{C}$, Grill $\mathrm{E}$, Winter $\mathrm{S}$, et al. Use of standardized assessment measures in acute inpatient rehabilitation. Physikalische Medizin Rehabilitationsmedizin Kurortmedizin. 2004; 14(1): p. 18-25.

3. Scott DA, Mills M, Black A, et al. Multidimensional rehabilitation programmes for adult cancer survivors. Cochrane Database Syst Rev. 2013; 2013(3): p. Cd007730.

4. Tennison JM, Fricke BC, Fu JB, et al. Medical Complications and Prognostic Factors for Medically Unstable Conditions During Acute Inpatient Cancer Rehabilitation. JCO Oncology Practice. 2021; [Epub ahead of print].

5. Centers for Medicare \& Medicaid Services. Evaluation and management services guide. 2021. https://www.cms.gov/Outreach-and-Education/ Medicare-Learning-Network-MLN/MLNProducts/Downloads/eval-mgmt-s erv-guide-ICN006764.pdf.

6. Effectively transferring patients to rehab: the essential guide to hospital-based care planning the essential guide to hospital-based care planning. Hospital Case Management. 2012: p. 123-4.

7. Alam E, Wilson RD, and Vargo MM. Inpatient cancer rehabilitation: a retrospective comparison of transfer back to acute care between patients with neoplasm and other rehabilitation patients. Am J Phys Med Rehabil. 2008; 89(7): p. 1284-1289

8. Asher A., Roberts PS, Bresee C, et al. Transferring inpatient rehabilitation facility cancer patients back to acute care (TRIPBAC). PM\&R. 2014; 6(9): p. 808-813.

9. Tennison JM, Rianon NJ, Manzano JG, et al. Thirty-day hospital readmission rate, reasons, and risk factors after acute inpatient cancer rehabilitation. Cancer Med. 2021. [Epub ahead of print]. 